\title{
Review Article \\ Skewed Epigenetics: An Alternative Therapeutic Option for Diabetes Complications
}

\author{
Gabriele Togliatto, Patrizia Dentelli, and Maria Felice Brizzi \\ Department of Medical Sciences, University of Turin, Corso Dogliotti 14, 10126 Turin, Italy \\ Correspondence should be addressed to Maria Felice Brizzi; mariafelice.brizzi@unito.it
}

Received 23 December 2014; Revised 7 April 2015; Accepted 22 April 2015

Academic Editor: Jun Panee

Copyright (C) 2015 Gabriele Togliatto et al. This is an open access article distributed under the Creative Commons Attribution License, which permits unrestricted use, distribution, and reproduction in any medium, provided the original work is properly cited.

\begin{abstract}
Vascular complications are major causes of morbidity and mortality in type 2 diabetes patients. Mitochondrial reactive oxygen species (ROS) generation and a lack of efficient antioxidant machinery, a result of hyperglycaemia, mainly contribute to this problem. Although advances in therapy have significantly reduced both morbidity and mortality in diabetic individuals, diabetesassociated vascular complications are still one of the most challenging health problems worldwide. New healing options are urgently needed as current therapeutics are failing to improve long-term outcomes. Particular effort has recently been devoted to understanding the functional relationship between chromatin structure regulation and the persistent change in gene expression which is driven by hyperglycaemia and which accounts for long-lasting diabetic complications. A detailed investigation into epigenetic chromatin modifications in type 2 diabetes is underway. This will be particularly useful in the design of mechanismbased therapeutics which interfere with long-lasting activating epigenetics and improve patient outcomes. We herein provide an overview of the most relevant mechanisms that account for hyperglycaemia-induced changes in chromatin structure; the most relevant mechanism is called "metabolic memory."
\end{abstract}

\section{Introduction}

Diabetes is a leading cause of morbidity and mortality across the world $[1,2]$. Population age, obesity, and modern sedentary lifestyles mean that the incidence of type 2 diabetes (T2D) has significantly increased worldwide in recent years [2-4]. Type 2 diabetes is associated with long-term vascular complications, including endothelial dysfunction, atherosclerosis, nephropathy, retinopathy, and peripheral arterial disease (PAD) [5-8]. Although advances in therapy have led to a significant reduction in both micro- and macrovascular complications, overall cardiovascular risk is still a clinical problem [9-12]. The Diabetes Control and Complications Trial (DCCT/EDIC) $[9,10]$ and the UK Prospective Diabetes Study (UKPDS) [11, 12] have shown that a coupling of intensive glycaemic control and the close monitoring of both blood pressure and cholesterol may not be effective in protecting patients from vascular complications, thus suggesting that diabetes-associated cardiovascular risk factors may rely on the so-called "metabolic memory." "Metabolic memory" mainly reflects epigenetic changes driven by hyperglycaemiainduced oxidative stress [13-16]. Attention has so far been paid to dissecting these epigenetic mechanisms and defining a diabetes epigenetic signature which would allow new therapeutic approaches, which could "erase" epigenetics and improve patient outcomes, to be developed. This concise review provides a synopsis of how epigenetic mechanisms impact long-term diabetes-associated complications.

\section{Epigenetic Changes: ROS-Mediated Effects in Diabetes}

Free radical accumulation in the vasculature of diabetic patients is considered to be the most significant mechanism in long-lasting vascular complications $[17,18]$. The key role played by mitochondrial ROS generation is a key pathway 
in the natural history of diabetes-associated vascular disease and has been documented by the seminal studies of Brownlee and colleagues [18-21]. Similarly, data provided by El-Osta and colleagues have contributed to current knowledge that links ROS of mitochondrial origin to endothelial dysfunction [22-24]. More recently, the intimate association between increased oxidative stress of mitochondrial origin and defective antioxidant machinery has been used to explain pathophysiological pathways associated with a prolonged response even to transient hyperglycaemia exposure [16, 25]. The fact that this hyperglycaemic environment can be remembered by the vasculature, first suggested by Roy and colleagues in 1990 [26], has recently furnished the mechanistic elucidation of what is called "metabolic memory" As a matter of fact "metabolic memory" refers to epigenetic changes driven by hyperglycaemia-mediated mitochondrial ROS production. The term "epigenetic" was first coined by Waddington in 1942 to explain how environmental factors can affect the phenotype without altering the genotype. Although its conceptual origins date back to Aristotle (384322 BC), who believed in "epigenesis" in the development of an individual organic form from the unformed, traces of epigenetics were seen in the literature by the mid-nineteenth century. It currently refers to heritable traits that do not match changes in the DNA sequence. This concept has recently been cited as a means to explain how the complex interaction between genotype and environmental cues (hyperglycaemia in diabetes) brings about "metabolic memory" [13-16, 2225]. Long- (heritable) or short-acting (nonheritable) environmental cues can lead to long- or short-term epigenetic effects, respectively. In diabetes, both mechanisms can take part in reprogramming the epigenome and eventually give details about the awful "metabolic memory" of postprandial hyperglycaemia episodes $[14,15,27]$. Over the last few years, a number of studies have shown that chromatin modification, in the nucleus, and microRNA (miR) deregulation, in the cytoplasm, are hallmarks of the long-lasting detrimental effects of hyperglycaemia which may translate into the high residual cardiovascular risk that diabetic patients face despite multifactor intervention [28].

\section{Epigenetics in the Nucleus}

Chromatin is the form in which the nucleic acids are found in the cell. Chromatin is, in fact, a macromolecular complex formed of DNA, proteins (histones), and RNA. Gene transcription is strictly controlled by the dynamic arrangement of chromatin: transcriptionally active (euchromatin) and transcriptionally inactive (heterochromatin). Histone posttranslational modifications (PTHMs) and DNA methylation both control gene expression. Changes in chromatin structure as a result of an imbalance in ROS production is the most relevant nuclear epigenetic mechanism and accounts for the longlasting effects of hyperglycaemia [29-31]. Histones and DNA are considered to be the foremost targets of epigenetic factors in this setting [29-31]. PTHM modification alters chromatin packing and results in different DNA arrangements and readouts. The most relevant PTHM changes in diabetes are lysine acetylation, by histone acetyltransferases (HATs), and lysine and arginine methylation, by histone methyltransferases (HMTs) [32]. The acetylation of $\mathrm{H} 3 \mathrm{~K}$ and $\mathrm{H} 4 \mathrm{~K}$ terminal tails generally erases any interaction with DNA, allowing euchromatin to form and gene transcription to occur. Conversely, histone methylation is associated with a more complex and "capricious" gene transcriptional status [13-16]. Indeed, the mono-, di-, or trimethylation of various lysine residues in the $\mathrm{H} 3$ tail can cause either transcriptional activation or repression. Of the enzymes that regulate this process, histone methyltransferases Set7 and Set9 are the most relevant, are activated in the hyperglycaemic settings, and mainly control genes involved in the inflammatory response [33]. Nevertheless, as a result of histone acetylation/methylation, a number of inflammatory genes undergo transcription in response to hyperglycaemia over time $[14,16,28]$. This sustains the relevance of such a mechanism in perpetuating tissue damaging signals in diabetes. Even transient hyperglycaemia can lead to long-lasting epigenetic changes in the promoter of the NF- $\kappa \mathrm{B}-\mathrm{p} 65$ gene, resulting in the increased expression of its product in the diabetic setting $[22,34]$. More recently, it has been reported that the mitochondrial adaptor protein p $66^{\text {shc }}$ may be transcriptionally regulated by $\mathrm{H} 3$ acetylation [25]. p $66^{\text {shc }}$ overexpression and mitochondrial translocation have been linked to increased mitochondrial ROS generation, reduced nitric oxide bioavailability, and vascular cell apoptosis [25]. The biological relevance of $\mathrm{H} 3$ acetylation in p $66^{\text {shc }}$ expression was further supported by the observation that the overexpression of one member of class III histone deacyltransferase (HDAC) SIRT1 inhibits hyperglycaemiainduced $\mathrm{p} 66^{\text {shc }}$ expression, improves endothelial function, and reduces oxidative stress markers [35] A close connection between $\mathrm{p} 66^{\text {shc }}$, SIRT1, and tumour transcription factor $\mathrm{p} 53$ $[25,36,37]$ has been also reported, which supports the crucial role that the epigenetic mechanism plays in dictating cell fate, predominantly in the vascular system. $\mathrm{p} 66^{\text {shc }}$ is a good example of a nuclear epigenetic target as its expression can be regulated by DNA methylation as well [25]. DNA methylation depends on three different DNA methyltransferases (DMT1, DM3A, and DM3B) in mammalian cells [38]. In these cells, DMT3A and DMT3B provide de novo methylation of the CpG dinucleotides on cytosine [39]. Therefore, DNA regions with increased CpG clusters, also called "CpG islands," dictate gene transcription status $[40,41]$. The hypermethylation of $\mathrm{CpG}$ islands in the promoter region commonly translates into transcription repression, while low methylation has the opposite effect [42]. The fact that DNA methylation can influence the expression of genes involved in diabetesassociated complications has been documented in preclinical models of nephropathy and retinopathy $[43,44]$. In addition, genome-wide methylation studies indicate that 19 prospective $\mathrm{CpG}$ regions are associated with an increased risk of diabetic nephropathy in type 1 diabetic patients [45]. Finally, studies performed on peripheral blood cells recovered from patients participating in the DCCT/EDIC study have demonstrated a correlation between chronic complications and $\mathrm{H} 3 \mathrm{~K} 9$ acetylation [46]. 


\section{Epigenetics Regulated by Cytoplasmic Factors}

In addition to DNA methylation and histone modifications, microRNAs (miRs) have emerged as relevant epigenetic regulators. miRs are a class of noncoding sequences which regulate gene expression at the posttranscriptional level via the inhibition of ribosome function, resulting in the translational repression or degradation of the target mRNA [47]. Although miRs most commonly lead to decreased gene expression, they can also induce gene upregulation by negatively modulating the expression of inhibitory genes. However, miR downregulation may also upregulate previously suppressed genes [4749]. More importantly, single miR targets multiple genes while single genes can be regulated by several miRs [47-49]. A number of studies demonstrating that miR expression is tissue specific have suggested that miRs may be important to establish and maintain cell type and tissue identity [4953]. However, in recent years their role in mediating chronic diseases has been deeply investigated. As a matter of fact, abnormal miR levels have been described in clinical and preclinical models of diabetes-associated complications [54, 55]. The role of miRs in diabetic nephropathy (DN) has been extensively studied. Altered levels of miR25- and miR21driven posttranscriptional regulation of the NADPH-oxidase subunits NOX4 and PTEN, respectively, have been linked to diabetic nephropathy in rat and mouse models $[56,57]$. Moreover, Wang and colleagues [58] demonstrated that the treatment of proximal-tubular epithelial cells with TGF- $\beta 1$ and TGF- $\beta 2$ led to miR-141 and miR-200a downregulation. The overexpression of miR-200a was found to be associated with the posttranscriptional regulation of TGF- $\beta 2$ which is consistent with miR-200a's role in the development and progression of TGF- $\beta$-dependent kidney fibrosis [58]. Similarly, increased miR-377 levels contributed to fibronectin production and kidney fibrosis in human and mouse mesangial cells that were exposed to TGF- $\beta$ and hyperglycemia [59]. Furthermore, kidney specific miR-192 knockdown was found to provide protection against diabetic nephropathyassociated kidney fibrosis [60].

Several miRs have been associated with the early stages of diabetic retinopathy (DR) development as well as disease progression. An evaluation of retinal endothelial cells and diabetic retinas indicated that numerous different targets were involved in angiogenesis, inflammation, and oxidative stress. Feng et al. [61] demonstrated that both endothelial cells from large vessels and retinal microvessels that had been treated with high glucose content express high fibronectin (FN) levels by means of miR-146a downregulation. Moreover, they also show that miR-200b controls miR-146a and FN expression, via the histone acetylator p300, in hyperglycemic conditions not only in the retina but also in the heart and kidney.

Recent data indicate that the reality is more complicated than expected as other miRs are involved in HDAC regulation [62]. Indeed, it has been reported that miR-29 and miR206 overexpression leads to HDAC4 translational repression during myogenic differentiation [63]. Furthermore, Lin et al. [64] demonstrated that hyperglycemia contributes to nephrin acetylation and renal dysfunction by impairing miR-29a signaling which resulted in improved HDAC4 action. Various miRs, including miR1, miR206, miR133a, miR221/222, and miR126, have also been implicated in cardiovascular complications as they act on various targets in cardiomyocytes and vascular cells [17, 65-67], while cardiac microvascular endothelial cells were found to express high miR-320 levels in a preclinical model of type 2 diabetes [68].

Although not as intensely investigated, unbalanced ROS production was found to be involved in miR deregulation [69]. In this regard, we have recently reported that interfering with mechanisms involved in ROS production rescues miR126 expression and reverts the epigenetic pattern in endothelial cells [70]. Indeed, we were able to reprogram some epigenetic abnormalities, such as SIRT1 expression and p53 and H3K56 acetylation status, in ob/ob mice subjected to peripheral artery disease by administering the naturally occurring hormone, unacylated ghrelin (UnAG) [70]. As this effect translates into hind limb function improvement, the notion of epigenome reprogramming being able to offer clinical benefits in a diabetic setting is strengthened.

\section{An Epigenomic Fingerprint for Identifying High-Risk Diabetic Patients}

As stated above, the epigenome refers to DNA methylation, histone modification, and chromatin accessibility throughout the genome. Each cell type possesses a unique epigenome, which defines its regulatory program, and each individual displays unique epigenome modifications [71, 72]. It has been extensively demonstrated that exposure to environmental cues affects an individual via their own epigenome. It has therefore become clear that an understanding of how genetic, epigenetic, and environmental factors interact with each other to drive chronic disease development and progression is mandatory $[14,15,27,28,73]$. Advances in genome-wide technologies and bioinformatics have opened up new possibilities in recent years $[74,75]$. The use of chipsequencing to map several histone and DNA methylation marks has demonstrated that changes in DNA methylation patterns and histone modification are crucial for genetic information readout [76]. A genome-wide map of epigenetic modifications across several pathologic conditions, including diabetes, would therefore be particularly relevant in therapeutic target identification. The Human Epigenome Project, established in 1999, identifies and interprets genome-wide DNA methylation patterns in human genes. It is therefore widely expected that an exact and detailed map of epigenetic variations may allow scientists to better understand epigenetic biogenesis and function and further improve genebased treatment as well as preventing diabetic complications. In this sense, epigenomic analysis in human pancreatic islets shows a strict association with gene expression and histone modification and is possibly relevant to diabetes [77]. More recently, data from Nilsson et al. [78] have provided support for the role played by DNA methylation in modulating the expression of genes associated with discrete pathways that are causally involved in T2D, thus strengthening 


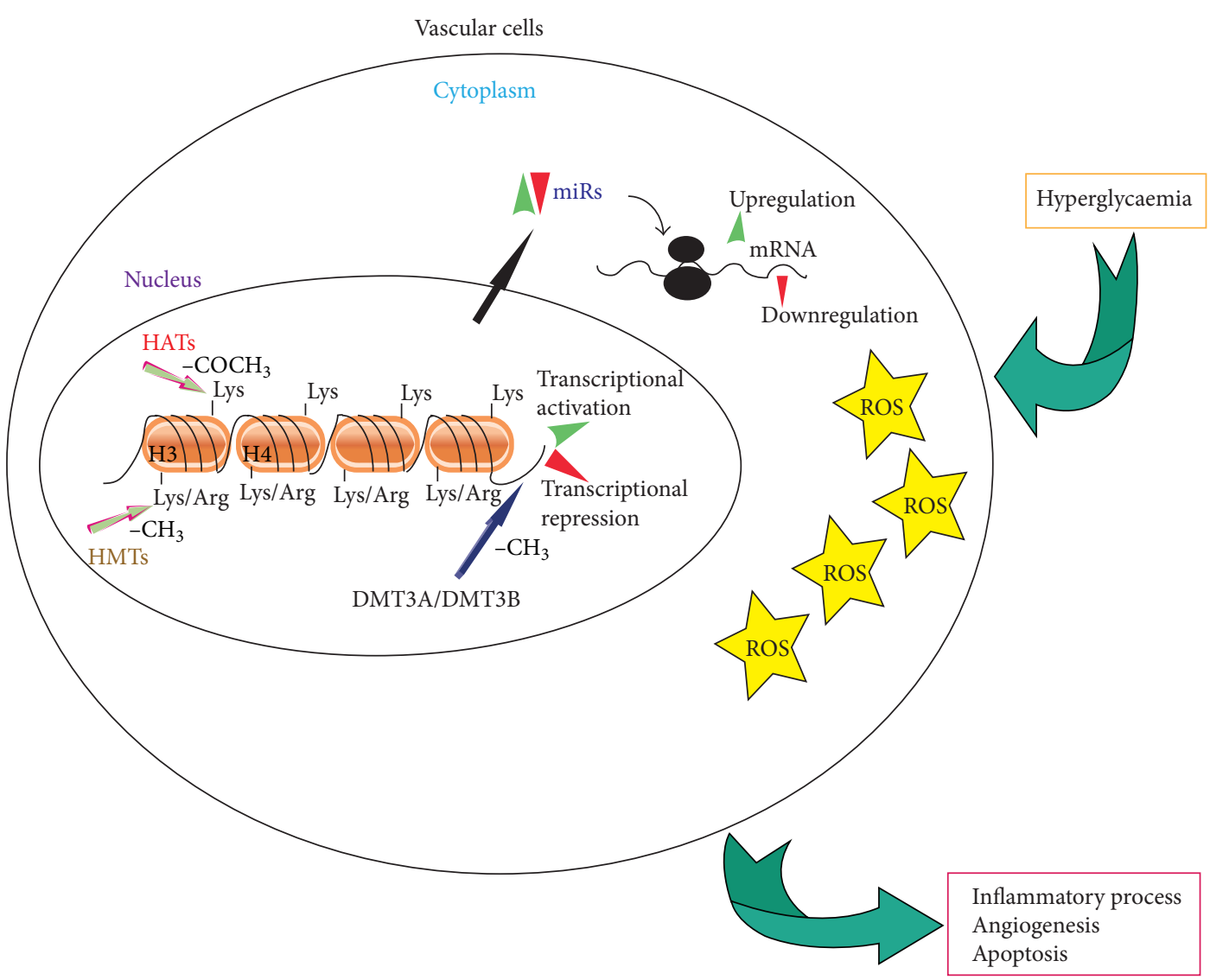

FIGURE 1: Schematic representation of epigenetic mechanisms in diabetic setting. In the nucleus ROS-mediated histone acetylation/methylation as well as DNA methylation accounts for gene transcriptional activation or repression. In the cytoplasm ROS-mediated changes in miR expression drive posttranscriptional regulation of target genes. Such epigenetic changes impact long-term diabetes-associated complications. HTAs: histone acetyltransferases; HMTs: histone methyltransferases; DMT: DNA methyltransferase; ROS: reactive oxygen species; miRs: microRNAs; Lys: lysine; Arg: arginine; $-\mathrm{COCH}_{3}$ : acetyl radical; $-\mathrm{CH}_{3}$ : methyl radical.

the notion that genetics, epigenetics, and environment cues may also mutually contribute to type 2 diabetes susceptibility. A challenge for the future thus lies in using next-generation sequencing techniques, such as RNA-sequencing and wholegenome sequencing, to identify the gene-expression regulatory network associated with high-risk diabetic patients on the DNA level.

\section{Pharmacological Reprogramming of the Epigenome: A Novel Therapeutic Option}

A detailed characterization of deranged gene expression patterns in diabetes has pulled clinicians towards epigenetic reprogramming approaches. A number of preclinical studies have indicated that gene expression can be modulated by treatment which interferes with the acetylation and methylation of histone/DNA complexes $[16,28]$. As a matter of fact, folate treatment can affect the expression of genes regulated by $\mathrm{CpG}$ methylation by reducing homocysteine [79]. p $66^{\text {shc }}$ is one of the genes regulated by CpG methylation in response to homocysteine [80]. A complete exploration of the potential clinical applications of folate is therefore an interesting challenge for the future. Other therapeutic epigenome-based options have been proposed; however, potential clinical application is still being debated. A reason for this was the failure of resveratrol, which was originally described as a potent SIRT1 activator that was able to rescue NO bioavailability [81] and increase $\mathrm{p} 66^{\text {shc }}$ promoter deacetylation [35], to provide beneficial effects when supplemented in obese patients [82]. A number of preclinical studies have also been performed on metformin, which is currently used to treat diabetic patients and which is known to increase SIRT1 expression and suppress NF- $\kappa \mathrm{B}$ activation [37]. Indeed, it has been shown that metformin is effective in suppressing the deleterious effects of hyperglycaemia in retinal capillary endothelial cells and in the retinas of diabetic animals by modulating the SIRT-1/LKB1/AMPK pathway [37]. However, the early enthusiasm for this drug that acts in the metabolism is in stark contrast with the controversial results obtained in UKPDS and Action to Control Cardiovascular Risk in Diabetes (ACCORD) trials, where metformin treatment led to reduced cardiovascular events in the first and an increased occurrence of these events in the second. Finally, the use of curcumin, known to exert beneficial effects by acting on the histone acetylator p300, has been proposed. However, even 
curcumin failed to be successful and the authors suggest that curcumin finds use as an adjuvant therapy in type 2 diabetic patients [83].

While the removal of epigenetic tags appears to be crucial in improving diabetes patient outcomes, clinical study results are far from convincing. It is, however, always worth remembering that the number of trials and the patients included in them is still a source of major bias when attempting to obtain persuasive results. This implies that effort should be made to assess the "true" clinical efficacy of the above compounds in randomized controlled trials. Moreover, genome-wide analysis of DNA methylation and histone modifications may well be the tool of the future when exploring new "personalized signature" based therapeutics.

\section{Conclusions and Perspectives}

Increased ROS of mitochondrial origin and defective mitochondrial electron transfer chains appear to be the most relevant mechanisms in what is known as "metabolic memory." "Metabolic memory," which refers to the effects of hyperglycaemia on long-term vascular complications, mainly relies on changes in chromatin structures and miR expression (Figure 1) [28]. Over the last few years, more details on how hyperglycaemia drives changes in the epigenome have been provided and a putative epigenome signature has been proposed. Moreover, genome-wide methylation studies have indicated that epigenetic mechanisms can predict nephropathy in type 1 diabetic patients [45]. Thus, future efforts should be directed towards clinically exploiting the epigenome signature so as to identify patients with a high risk of complications and epigenome targeting approaches. Indeed, recent efforts have been directed towards developing agents which are able to reprogram the epigenome and hamper the vicious hyperglycaemia driven circle which leads to residual cardiovascular risk in diabetic patients [84]. However, it is always worth remembering that since environmental factors are the main triggers of epigenetic modifications, a suitable lifestyle should be recommended above all other factors.

\section{Conflict of Interests}

The authors declare that there is no conflict of interests regarding the publication of this paper.

\section{Acknowledgments}

This work was supported by grants for Maria Felice Brizzi from Unito-Compagnia S. Paolo and from Fondazione per la Ricerca Diabetologica FO.Ri.SID.

\section{References}

[1] S. Wild, G. Roglic, A. Green, R. Sicree, and H. King, "Global prevalence of diabetes: estimates for the year 2000 and projections for 2030," Diabetes Care, vol. 27, no. 5, pp. 1047-1053, 2004.

[2] American Diabetes Association, "Diagnosis and classification of diabetes mellitus," Diabetes Care, vol. 35, pp. S64-S71, 2012.
[3] A. H. Mokdad, E. S. Ford, B. A. Bowman et al., "Prevalence of obesity, diabetes, and obesity-related health risk factors, 2001," Journal of the American Medical Association, vol. 289, no. 1, pp. 76-79, 2003.

[4] P. Hossain, B. Kawar, and M. El Nahas, "Obesity and diabetes in the developing world-a growing challenge," The New England Journal of Medicine, vol. 356, no. 3, pp. 213-215, 2007.

[5] M. Gili, A. Orsello, S. Gallo, and M. F. Brizzi, "Diabetesassociated macrovascular complications: cell-based therapy a new tool?" Endocrine, vol. 44, no. 3, pp. 557-575, 2012.

[6] A. Trombetta, G. Togliatto, A. Rosso et al., "Increase of palmitic acid concentration impairs endothelial progenitor cell and bone marrow-derived progenitor cell bioavailability: role of the STAT5/PPAR $\gamma$ transcriptional complex," Diabetes, vol. 62, no. 4, pp. 1245-1257, 2013.

[7] M. F. Brizzi, P. Dentelli, M. Pavan et al., "Diabetic LDL inhibits cell-cycle progression via STAT5B and p21 $1^{\text {waf }}$," Journal of Clinical Investigation, vol. 109, no. 1, pp. 111-119, 2002.

[8] F. Paneni, S. Costantino, and F. Cosentino, "Molecular mechanisms of vascular dysfunction and cardiovascular biomarkers in type 2 diabetes," Cardiovascular Diagnosis and Therapy, vol. 4, no. 4, pp. 324-332, 2014.

[9] "The effect of intensive treatment of diabetes on the development and progression of long-term complications in insulindependent diabetes mellitus. The Diabetes Control and Complications Trial Research Group," The New England Journal of Medicine, vol. 329, pp. 977-986, 1993.

[10] Writing Team for the Diabetes Control and Complications Trial/Epidemiology of Diabetes Interventions and Complications Research Group, "Sustained effect of intensive treatment of type 1 diabetes mellitus on development and progression of diabetic nephropathy: the Epidemiology of Diabetes Interventions and Complications (EDIC) study," Journal of the American Medical Association, vol. 290, no. 16, pp. 2159-2167, 2003.

[11] R. Turner, "Intensive blood-glucose control with sulphonylureas or insulin compared with conventional treatment and risk of complications in patients with type 2 diabetes (UKPDS 33). UK Prospective Diabetes Study (UKPDS) Group," The Lancet, vol. 352, no. 9131, pp. 837-853, 1998.

[12] R. R. Holman, S. K. Paul, M. A. Bethel, D. R. Matthews, and H. A. W. Neil, "10-Year follow-up of intensive glucose control in type 2 diabetes," The New England Journal of Medicine, vol. 359, no. 15, pp. 1577-1589, 2008.

[13] A. Ceriello, "Hypothesis: the 'metabolic memory', the new challenge of diabetes," Diabetes Research and Clinical Practice, vol. 86, supplement 1, pp. S2-S6, 2009.

[14] M. E. Cooper and A. El-Osta, "Epigenetics: mechanisms and implications for diabetic complications," Circulation Research, vol. 107, no. 12, pp. 1403-1413, 2010.

[15] M. A. Reddy and R. Natarajan, "Epigenetic mechanisms in diabetic vascular complications," Cardiovascular Research, vol. 90, no. 3, pp. 421-429, 2011.

[16] F. Paneni, M. Volpe, T. F. Lüscher, and F. Cosentino, "SIRT1, p $66^{\text {Shc }}$, and set7/9 in vascular hyperglycemic memory: bringing all the strands together," Diabetes, vol. 62, no. 6, pp. 1800-1807, 2013.

[17] G. Togliatto, A. Trombetta, P. Dentelli, A. Rosso, and M. F. Brizzi, "MIR221/MIR222-driven post-transcriptional regulation of P27KIP1 and P57KIP2 is crucial for high-glucose- and AGE-mediated vascular cell damage," Diabetologia, vol. 54, no. 7, pp. 1930-1940, 2011. 
[18] F. Giacco and M. Brownlee, "Oxidative stress and diabetic complications," Circulation Research, vol. 107, no. 9, pp. 10581070, 2010.

[19] T. Nishikawa, D. Edelstein, X. L. Du et al., "Normalizing mitochondrial superoxide production blocks three pathways of hyperglycaemic damage," Nature, vol. 404, no. 6779, pp. 787790, 2000.

[20] S. Kiritoshi, T. Nishikawa, K. Sonoda et al., "Reactive oxygen species from mitochondria induce cyclooxygenase-2 gene expression in human mesangial cells: potential role in diabetic nephropathy," Diabetes, vol. 52, no. 10, pp. 2570-2577, 2003.

[21] M. T. Coughlan, D. R. Thorburn, S. A. Penfold et al., "Rageinduced cytosolic ROS promote mitochondrial superoxide generation in diabetes," Journal of the American Society of Nephrology, vol. 20, no. 4, pp. 742-752, 2009.

[22] A. El-Osta, D. Brasacchio, D. Yao et al., "Transient high glucose causes persistent epigenetic changes and altered gene expression during subsequent normoglycemia," The Journal of Experimental Medicine, vol. 205, pp. 2409-2417, 2008.

[23] J. Okabe, C. Orlowski, A. Balcerczyk et al., "Distinguishing hyperglycemic changes by set7 in vascular endothelial cells," Circulation Research, vol. 110, no. 8, pp. 1067-1076, 2012.

[24] S. P. Gray, E. Di Marco, J. Okabe et al., "NADPH oxidase 1 plays a key role in diabetes mellitus-accelerated atherosclerosis," Circulation, vol. 127, no. 18, pp. 1888-1902, 2013.

[25] F. Paneni, P. Mocharla, A. Akhmedov et al., "Gene silencing of the mitochondrial adaptor $\mathrm{p} 66^{\text {Shc }}$ suppresses vascular hyperglycemic memory in diabetes," Circulation Research, vol. 111, no. 3, pp. 278-289, 2012.

[26] S. Roy, R. Sala, E. Cagliero, and M. Lorenzi, “Overexpression of fibronectin induced by diabetes or high glucose: phenomenon with a memory," Proceedings of the National Academy of Sciences of the United States of America, vol. 87, no. 1, pp. 404-408, 1990.

[27] L. Pirola, A. Balcerczyk, J. Okabe, and A. El-Osta, "Epigenetic phenomena linked to diabetic complications," Nature Reviews Endocrinology, vol. 6, no. 12, pp. 665-675, 2010.

[28] F. Paneni, S. Costantino, M. Volpe, T. F. Lüscher, and F. Cosentino, "Epigenetic signatures and vascular risk in type 2 diabetes: a clinical perspective," Atherosclerosis, vol. 230, no. 2, pp. 191-197, 2013.

[29] A. Bird, "DNA methylation patterns and epigenetic memory," Genes and Development, vol. 16, no. 1, pp. 6-21, 2002.

[30] D. P. Bartel, "MicroRNAs: genomics, biogenesis, mechanism, and function," Cell, vol. 116, no. 2, pp. 281-297, 2004.

[31] R. B. Meagher and K. J. Müssar, "The influence of DNA sequence on epigenome-induced pathologies," Epigenetics \& Chromatin, vol. 5, no. 1, article 11, 2012.

[32] M. Wegner, D. Neddermann, M. Piorunska-Stolzmann, and P. P. Jagodzinski, "Role of epigenetic mechanisms in the development of chronic complications of diabetes," Diabetes Research and Clinical Practice, vol. 105, pp. 164-175, 2014.

[33] P. Mathiyalagan, S. T. Keating, X. Du, and A. El-Osta, "Chromatin modifications remodel cardiac gene expression," Cardiovascular Research, vol. 103, no. 1, pp. 7-16, 2014.

[34] D. Brasacchio, J. Okabe, C. Tikellis et al., "Hyperglycemia induces a dynamic cooperativity of histone methylase and demethylase enzymes associated with gene-activating epigenetic marks that coexist on the lysine tail," Diabetes, vol. 58, no. 5, pp. 1229-1236, 2009.

[35] S. Zhou, H.-Z. Chen, Y.-Z. Wan et al., "Repression of P66Shc expression by SIRT1 contributes to the prevention of hyperglycemia-induced endothelial dysfunction," Circulation Research, vol. 109, no. 6, pp. 639-648, 2011.

[36] C.-S. Kim, S.-B. Jung, A. Naqvi et al., "P53 impairs endotheliumdependent vasomotor function through transcriptional upregulation of P66shc," Circulation Research, vol. 103, no. 12, pp. 1441-1450, 2008.

[37] Z. Zheng, H. Chen, J. Li et al., "Sirtuin 1-mediated cellular metabolic memory of high glucose via the LKB1/AMPK/ROS pathway and therapeutic effects of metformin," Diabetes, vol. 61, no. 1, pp. 217-228, 2012.

[38] S. K. T. Ooi and T. H. Bestor, "The colorful history of active DNA demethylation," Cell, vol. 133, no. 7, pp. 1145-1148, 2008.

[39] C. P. Walsh and T. H. Bestor, "Cytosine methylation and mammalian development," Genes and Development, vol. 13, no. 1, pp. 26-34, 1999.

[40] G. Liang, M. F. Chan, Y. Tomigahara et al., "Cooper activity between DNA methyltransferases in the maintenance methylation of repetitive elements," Molecular and Cellular Biology, vol. 22, no. 2, pp. 480-491, 2002.

[41] R. Straussman, D. Nejman, D. Roberts et al., "Developmental programming of $\mathrm{CpG}$ island methylation profiles in the human genome," Nature Structural and Molecular Biology, vol. 16, no. 5, pp. 564-571, 2009.

[42] S. Fan and X. Zhang, "CpG island methylation pattern in different human tissues and its correlation with gene expression," Biochemical and Biophysical Research Communications, vol. 383, no. 4, pp. 421-425, 2009.

[43] K. T. Williams, T. A. Garrow, and K. L. Schalinske, "Type I diabetes leads to tissue-specific DNA hypomethylation in male rats," Journal of Nutrition, vol. 138, no. 11, pp. 2064-2069, 2008.

[44] S. Tewari, Q. Zhong, J. M. Santos, and R. A. Kowluru, "Mitochondria DNA replication and DNA methylation in the metabolic memory associated with continued progression of diabetic retinopathy," Investigative Ophthalmology \& Visual Science, vol. 53, no. 8, pp. 4881-4888, 2012.

[45] C. G. Bell, A. E. Teschendorff, V. K. Rakyan, A. P. Maxwell, S. Beck, and D. A. Savage, "Genome-wide DNA methylation analysis for diabetic nephropathy in type 1 diabetes mellitus," BMC Medical Genomics, vol. 3, article 33, 2010.

[46] F. Miao, Z. Chen, S. Genuth et al., "Evaluating the role of epigenetic histone modifications in the metabolic memory of type 1 diabetes," Diabetes, vol. 63, no. 5, pp. 1748-1762, 2014.

[47] J. Krol, I. Loedige, and W. Filipowicz, "The widespread regulation of microRNA biogenesis, function and decay," Nature Reviews Genetics, vol. 11, no. 9, pp. 597-610, 2010.

[48] C. Urbich, A. Kuehbacher, and S. Dimmeler, "Role of microRNAs in vascular diseases, inflammation, and angiogenesis," Cardiovascular Research, vol. 79, no. 4, pp. 581-588, 2008.

[49] W. Filipowicz, S. N. Bhattacharyya, and N. Sonenberg, "Mechanisms of post-transcriptional regulation by microRNAs: are the answers in sight?" Nature Reviews Genetics, vol. 9, no. 2, pp. 102114, 2008.

[50] Y. Liang, D. Ridzon, L. Wong, and C. Chen, "Characterization of microRNA expression profiles in normal human tissues," $B M C$ Genomics, vol. 8, article 166, 2007.

[51] I. Alvarez-Garcia and E. A. Miska, "MicroRNA functions in animal development and human disease," Development, vol. 132, no. 21, pp. 4653-4662, 2005.

[52] A. Ribeiro, C. Schoof, A. Izzotti, L. Pereira, and L. Vasques, "MicroRNAs: modulators of cell identity, and their applications in tissue engineering," MicroRNA, vol. 3, no. 1, pp. 45-53, 2014. 
[53] M. R. Fabian, N. Sonenberg, and W. Filipowicz, "Regulation of mRNA translation and stability by microRNAs," Annual Review of Biochemistry, vol. 79, pp. 351-379, 2010.

[54] M. Kato, N. E. Castro, and R. Natarajan, "MicroRNAs: potential mediators and biomarkers of diabetic complications," Free Radical Biology and Medicine, vol. 64, pp. 85-94, 2013.

[55] M. Kato and R. Natarajan, "Diabetic nephropathy-emerging epigenetic mechanisms," Nature Reviews Nephrology, vol. 10, no. 9, pp. 517-530, 2014.

[56] Y. Fu, Y. Zhang, Z. Wang et al., "Regulation of NADPH oxidase activity is associated with miRNA-25-mediated NOX4 expression in experimental diabetic nephropathy," The American Journal of Nephrology, vol. 32, no. 6, pp. 581-589, 2010.

[57] Z. Zhang, H. Peng, J. Chen et al., "MicroRNA-21 protects from mesangial cell proliferation induced by diabetic nephropathy in db/db mice," FEBS Letters, vol. 583, no. 12, pp. 2009-2014, 2009.

[58] B. Wang, P. Koh, C. Winbanks et al., "miR-200a prevents renal fibrogenesis through repression of TGF- $\beta 2$ expression," Diabetes, vol. 60, no. 1, pp. 280-287, 2011.

[59] Q. Wang, Y. Wang, A. W. Minto et al., "MicroRNA-377 is upregulated and can lead to increased fibronectin production in diabetic nephropathy," The FASEB Journal, vol. 22, no. 12, pp. 4126-4135, 2008.

[60] S. Putta, L. Lanting, G. Sun, G. Lawson, M. Kato, and R. Natarajan, "Inhibiting microRNA-192 ameliorates renal fibrosis in diabetic nephropathy," Journal of the American Society of Nephrology, vol. 23, no. 3, pp. 458-469, 2012.

[61] B. Feng, S. Chen, K. McArthur et al., "miR-146a-mediated extracellular matrix protein production in chronic diabetes complications," Diabetes, vol. 60, no. 11, pp. 2975-2984, 2011.

[62] S. Swierczynski, E. Klieser, R. Illig, B. Alinger-Scharinger, T. Kiesslich, and D. Neureiter, "Histone deacetylation meets miRNA: epigenetics and post-transcriptional regulation in cancer and chronic diseases," Expert Opinion on Biological Therapy, vol. 15, no. 5, pp. 651-664, 2015.

[63] C. E. Winbanks, B. Wang, C. Beyer et al., "TGF-beta regulates miR-206 and miR-29 to control myogenic differentiation through regulation of HDAC4," The Journal of Biological Chemistry, vol. 286, no. 16, pp. 13805-13814, 2011.

[64] C. L. Lin, P. H. Lee, Y. C. Hsu et al., "MicroRNA-29a promotion of nephrin acetylation ameliorates hyperglycemia-induced podocyte dysfunction," Journal of the American Society of Nephrology, vol. 25, pp. 1698-1709, 2014.

[65] Z.-X. Shan, Q.-X. Lin, C.-Y. Deng et al., "MiR-1/miR-206 regulate Hsp60 expression contributing to glucose-mediated apoptosis in cardiomyocytes," FEBS Letters, vol. 584, no. 16, pp. 3592-3600, 2010.

[66] B. Feng, S. Chen, B. George, Q. Feng, and S. Chakrabarti, "miR133a regulates cardiomyocyte hypertrophy in diabetes," Diabetes/Metabolism Research and Reviews, vol. 26, no. 1, pp. 40-49, 2010.

[67] S. Meng, J.-T. Cao, B. Zhang, Q. Zhou, C.-X. Shen, and C.Q. Wang, "Downregulation of microRNA-126 in endothelial progenitor cells from diabetes patients, impairs their functional properties, via target gene Spred-1," Journal of Molecular and Cellular Cardiology, vol. 53, no. 1, pp. 64-72, 2012.

[68] X. H. Wang, R. Z. Qian, W. Zhang, S. F. Chen, H. M. Jin, and R. M. Hu, "MicroRNA-320 expression in myocardial microvascular endothelial cells and its relationship with insulin-like growth factor-1 in type 2 diabetic rats," Clinical and Experimental Pharmacology and Physiology, vol. 36, no. 2, pp. 181-188, 2009.
[69] A. Magenta, S. Greco, C. Gaetano, and F. Martelli, "Oxidative stress and microRNAs in vascular diseases," International Journal of Molecular Sciences, vol. 14, no. 9, pp. 17319-17346, 2013.

[70] G. Togliatto, A. Trombetta, P. Dentelli et al., "Unacylated ghrelin (UnAG) induces oxidative stress resistance in a glucose intolerance mouse model and peripheral artery disease by restoring endothelial cell miR-126 expression," Diabetes, vol. 64, no. 4, pp. 1370-1382, 2015.

[71] R. Margueron and D. Reinberg, "Chromatin structure and the inheritance of epigenetic information," Nature Reviews Genetics, vol. 11, no. 4, pp. 285-296, 2010.

[72] C. Alabert and A. Groth, "Chromatin replication and epigenome maintenance," Nature Reviews Molecular Cell Biology, vol. 13, no. 3, pp. 153-167, 2012.

[73] R. L. Jirtle and M. K. Skinner, "Environmental epigenomics and disease susceptibility," Nature Reviews Genetics, vol. 8, no. 4, pp. 253-262, 2007.

[74] N. Grarup, C. H. Sandholt, T. Hansen, and O. Pedersen, "Genetic susceptibility to type 2 diabetes and obesity: from genome-wide association studies to rare variants and beyond," Diabetologia, vol. 57, pp. 1528-1541, 2014.

[75] E. E. Ntzani and F. K. Kavvoura, "Genetic risk factors for type 2 diabetes: insights from the emerging genomic evidence," Current Vascular Pharmacology, vol. 10, no. 2, pp. 147-155, 2012.

[76] R. Lister, M. Pelizzola, R. H. Dowen et al., "Human DNA methylomes at base resolution show widespread epigenomic differences," Nature, vol. 462, no. 7271, pp. 315-322, 2009.

[77] R. Bhandare, J. Schug, J. Le Lay et al., "Genome-wide analysis of histone modifications in human pancreatic islets," Genome Research, vol. 20, no. 4, pp. 428-433, 2010.

[78] E. Nilsson, P. A. Jansson, A. Perfilyev et al., "Altered DNA methylation and differential expression of genes influencing metabolism and inflammation in adipose tissue from subjects with type 2 diabetes," Diabetes, vol. 63, no. 9, pp. 2962-2976, 2014.

[79] P. Yi, S. Melnyk, M. Pogribna, I. P. Pogribny, R. J. Hine, and S. J. James, "Increase in plasma homocysteine associated with parallel increases in plasma $S$-adenosylhomocysteine and lymphocyte DNA hypomethylation," The Journal of Biological Chemistry, vol. 275, no. 38, pp. 29318-29323, 2000.

[80] C.-S. Kim, Y.-R. Kim, A. Naqvi et al., "Homocysteine promotes human endothelial cell dysfunction via site-specific epigenetic regulation of p66shc," Cardiovascular Research, vol. 92, no. 3, pp. 466-475, 2011.

[81] D. M. Breen, V. W. Dolinsky, H. Zhang et al., "Resveratrol inhibits neointimal formation after arterial injury through an endothelial nitric oxide synthase-dependent mechanism," Atherosclerosis, vol. 222, no. 2, pp. 375-381, 2012.

[82] M. M. Poulsen, P. F. Vestergaard, B. F. Clasen et al., "Highdose resveratrol supplementation in obese men: an investigatorinitiated, randomized, placebo-controlled clinical trial of substrate metabolism, insulin sensitivity, and body composition," Diabetes, vol. 62, no. 4, pp. 1186-1195, 2013.

[83] M. A. El-Moselhy, A. Taye, S. S. Sharkawi, S. F. I. El-Sisi, and A. F. Ahmed, "The antihyperglycemic effect of curcumin in high fat diet fed rats. Role of TNF- $\alpha$ and free fatty acids," Food and Chemical Toxicology, vol. 49, no. 5, pp. 1129-1140, 2011.

[84] C. A. Hamm and F. F. Costa, "The impact of epigenomics on future drug design and new therapies," Drug Discovery Today, vol. 16, no. 13-14, pp. 626-635, 2011. 


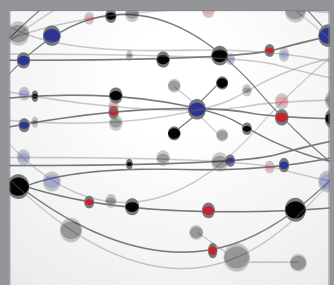

The Scientific World Journal
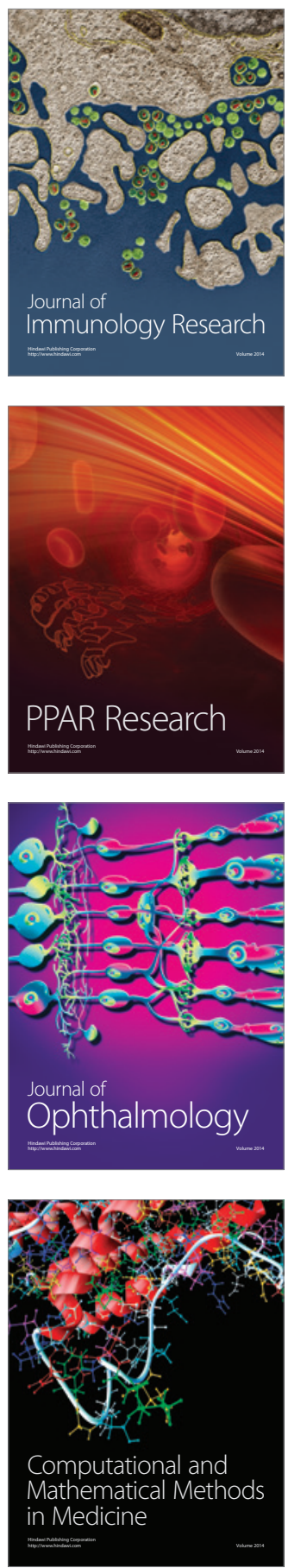

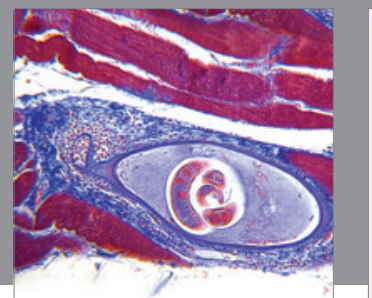

Gastroenterology

Research and Practice
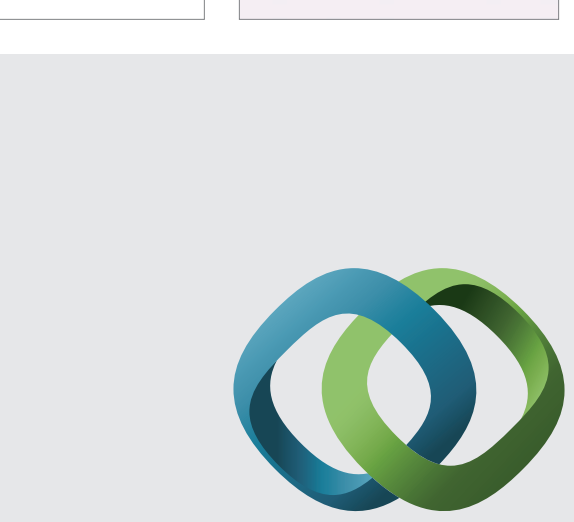

\section{Hindawi}

Submit your manuscripts at

http://www.hindawi.com
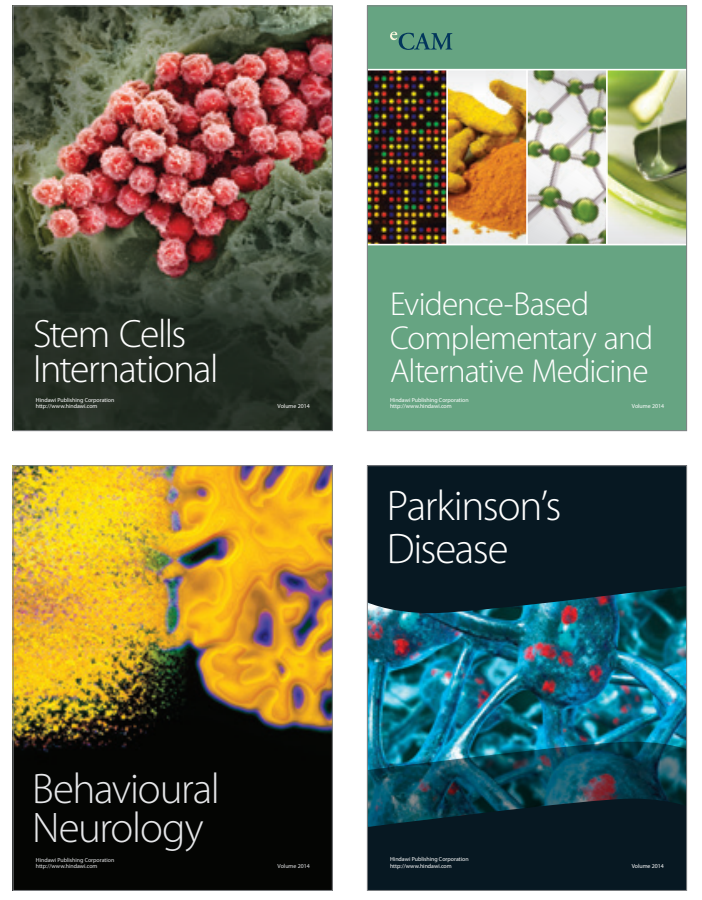
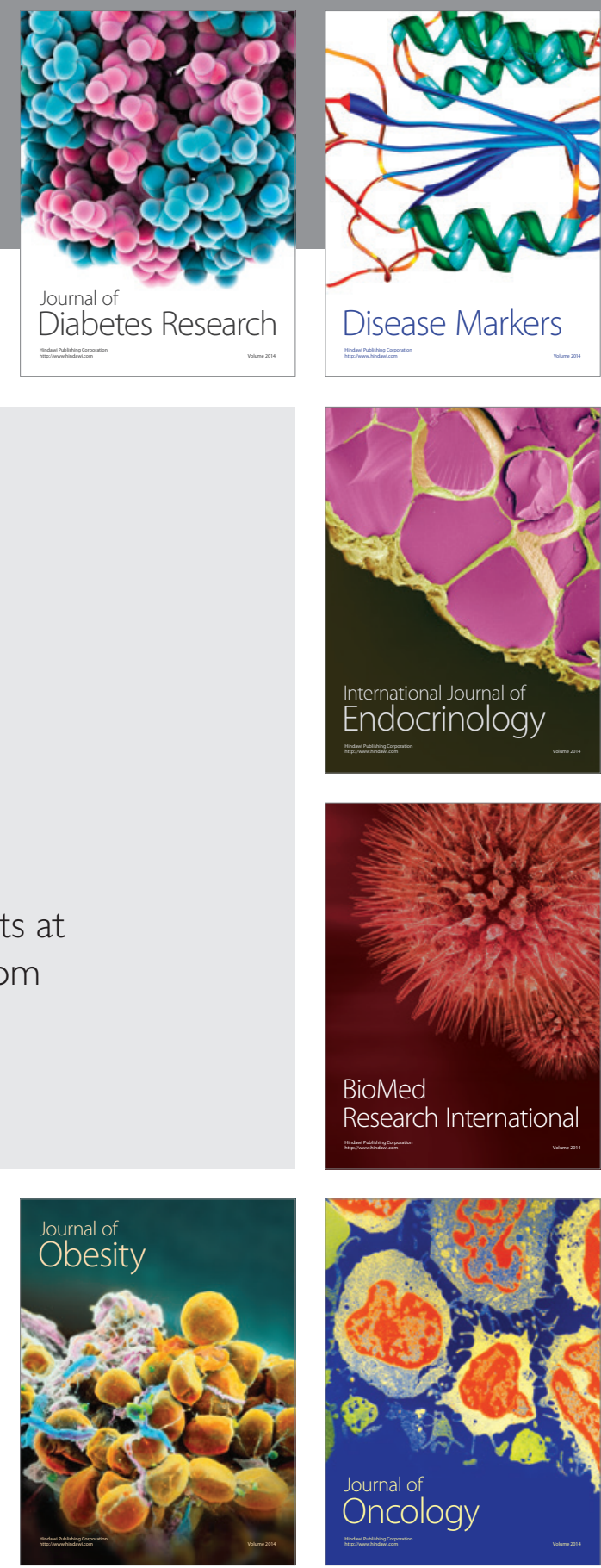

Disease Markers
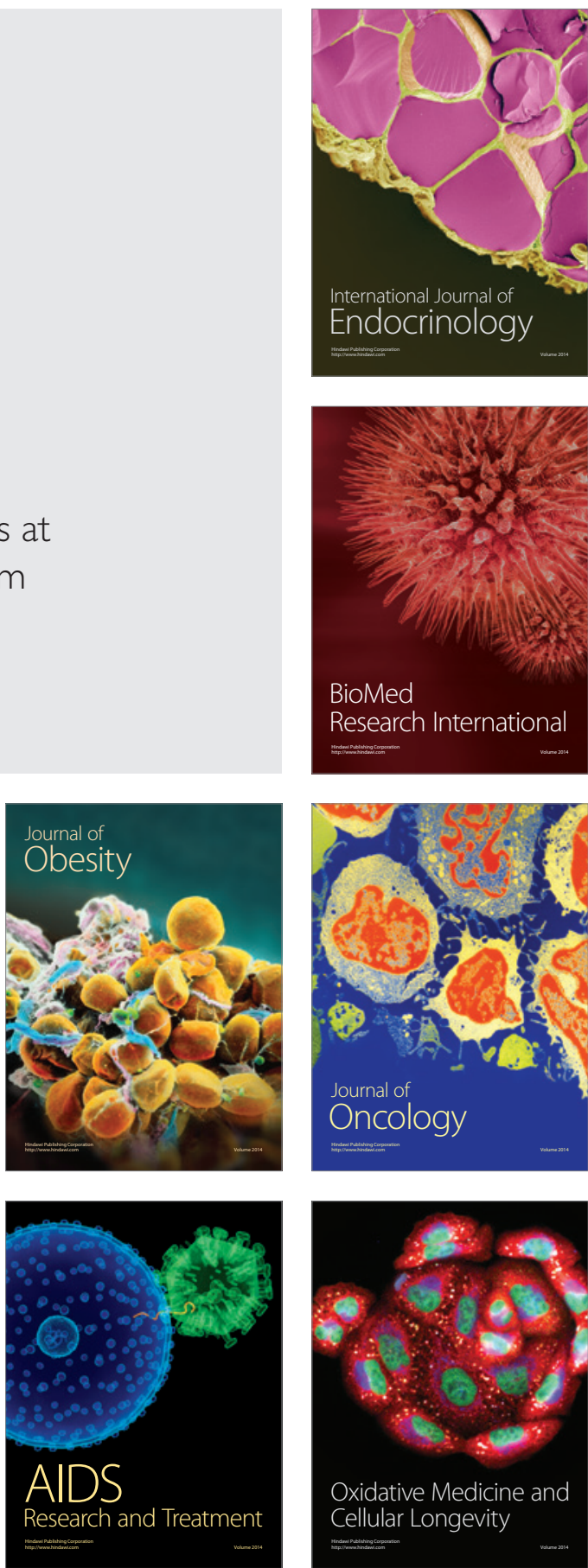